\title{
Towards real persons: Clinical judgement and philosophy of psychiatry
}

One of the motivations for the new philosophy of psychiatry is the need to understand changing ideas in mental health care. In the last century, changes in both physical and biological theory prompted work in philosophy of physics and philosophy of biology to understand those fields better, attempts which were continuous with empirical work. At the start of this century, changes in psychiatry promise increased interest in the philosophy of psychiatry as an attempt, alongside empirical research, to understand the conceptual underpinnings of mental heath care. While philosophical methods are distinct from empirical methods, the work is truly interdisciplinary, growing organically from the complexities of demand on psychiatric care and, although philosophical, carried out by philosophers and psychiatrists alike. One focus is the nature of clinical judgement in psychiatric diagnosis. In this short note I will briefly sketch some issues that arise from a current idea: that psychiatric diagnosis should include idiographic elements.

\section{Idiographic understanding and comprehensive diagnosis}

There has been a recent growth in emphasis on the importance of idiographic understanding in psychiatric diagnosis. The World Psychiatric Association (WPA) advocates the development of a 'comprehensive' model of diagnosis or assessment. A WPA workgroup charged with formulating an 'International Guidelines for Diagnostic Assessment' (IGDA) has published a guideline called 'Idiographic (Personalised) Diagnostic Formulation' which recommends an idiographic component within psychiatric diagnoses. If accepted, skilled psychiatric diagnostic judgement will have to include an idiographic as well as nomothetic component. What would this involve?

In psychology, the popular use of the distinction between idiographic and nomothetic was widely ascribed to the 
personality psychologist G W Allport. It is generally taken to refer to a distinction between qualitative research based on individual case studies and quantitative cohort-based research. But this way of drawing a distinction need not amount to a distinction of kind. The distinction between research on individuals and cohorts, for example, may not be significant if individuals are described in general terms.

If 'qualitative' is defined merely in opposition to mathematically encoded or statistical methods then, again, that need not correspond to any underlying methodological distinction. In geology, for example, both mathematical and non-mathematical descriptions are used depending on context, but this does not reflect any significant methodological differences.

The term 'idiographic' was first introduced, in a contrast with 'nomothetic', by the Kantian philosopher of science Wilhelm Windelband in 1894 in this way:

In their quest for knowledge of reality, the empirical sciences either seek the general in the form of the law of nature or the particular in the form of the historically defined structure. On the one hand, they are concerned with the form which invariably remains constant. On the other hand, they are concerned with the unique, immanently defined content of the real event. The former disciplines are nomological sciences. The latter disciplines are sciences of process or sciences of the event. The nomological sciences are concerned with what is invariably the case. The sciences of process are concerned with what was once the case. If I may be permitted to introduce some new technical terms, scientific thought is nomothetic in the former case and idiographic in the latter case' (Windelband' pp. 175-176).

Nomothetic approaches chart lawlike, or nomological, generalities. Idiographic understanding concerns individual cases described in non-general ways. Both are forms of empirical inquiry, both can deserve the label 'science' but the form of intelligibility is different. Windelband emphasises the idea that the distinction is not one of subject matter but method. The same subject matter might be treated by both approaches. Evolutionary history, for example, can be taken to manifest a constant underlying form (with repeatable effects) susceptible of nomothetic analysis or be thought of as a lengthy but single event, capable of idiographic historical understanding.

\section{What is different about idiographic understanding?}

So far, however, I have not specified the way in which idiographic understanding, which focuses on individuals, is supposed to be different. Windelband offers a number of unsatisfactory hints. It, for example, 'seeks structural forms' and 'is devoted to the faithful delineation of the particulars'1 (p. 178). But this does not distinguish idiographic understanding from a natural scientific account of particular chemical structures couched in invariant nomological terms.

Windelband also suggests that the task of idiographic understanding 'is to breathe new life into some structure of the past in such a way that all of its concrete and distinctive features acquire an ideal actuality or contemporaneity' (p. 178). But even vivid portrayal, or imaginative recreation, of individual events need not amount to understanding. A child might, for example, imaginatively recreate or re-enact the moves of a game of chess without understanding the rules or strategy.

The underlying appeal of idiographic understanding, both in Windelband's work and in its application in comprehensive diagnosis, is its contrast with general knowledge. Windelband argues that an over-emphasis on the general has been present in human thought since the Greeks:

'But the more we strive for knowledge of the concept and the law, the more we are obliged to pass over, forget, and abandon the singular fact as such ...

In opposition to this standpoint, it is necessary to insist upon the following: every interest and judgment, every ascription of human value is based upon the singular and the unique' (Windelband' pp. 181-1821.

Examining value judgements is supposed to reveal the fundamental importance of singular cases as opposed to general kinds in empirical judgements. This implies that there is an important role for a kind of judgement in which there is no implicit comparison - as there is with any general concept - with other cases. Such a judgement would be essentially particular and thus could not include general conceptual elements. It would aim to reflect the uniqueness of individuals for mental health care.

\section{Idiographic and still valid?}

This construal of idiographic suggests an immediate problem. How can an essentially one-off judgement meet a general aim of medical diagnosis: validity? What sense is there to the idea that a judgement that does not make implicit comparison to other cases is a valid judgement? In fact the idea that there can be judgements that do not depend on more general conceptual elements and thus are not formed in complete isolation from the rest of one's world view has come under fire in the philosophy of science in the idea that observation is always theory-dependent and more generally in the criticism of the 'Myth of the Given'. If these criticisms are correct then idiographic understanding is itself a myth. 
What then is, or should be, distinctive about comprehensive diagnosis? I suggest that it is the narrative element rather than the supposedly idiographic which is important. Narrative understanding contrasts with nomological or lawlike understanding or explanation not because it aims at one-off events but because it is normative. The connections between elements of a narrative account of a subject reflect how a subject ought to think and act. The subject is compared to an ideal of good thinking. This contrasts with subsumption of events under natural laws in which that normative dimension of what ought to happen is missing. Tailored to an understanding of persons, the elements of a narrative are mental states described in a rational pattern of propositional attitudes.

'The concepts of the propositional attitudes have their proper home in explanations of a special sort: explanations in which things are made intelligible by being revealed to be, or to approximate to being, as they rationally ought to be. This is to be contrasted with a style of explanation in which one makes things intelligible by representing their coming into being as a particular instance of how things generally tend to happen' (McDowell ${ }^{2}$ p.389).

One of the ways in which the distinction manifests itself is in the different way anomalies are accounted for in these two forms of understanding. If an explanation by subsumption under a natural law fails because observations of what have happened fail to fit the law then so much the worse either for the accuracy of observations or for the natural law. But this is not the case for narrative accounts where deviations from the standards of good reasoning can be accommodated as occasional lapses of judgement, perhaps explained by other aspects of the pattern which do accord with the standards. (One can, for example, form a mistaken belief for a good reason.)

Despite this difference, narrative accounts are nevertheless couched in general terms and consequently there is no tension between adding such elements to comprehensive diagnosis and aiming for the validity of psychiatric diagnosis. Thus if the supposedly idiographic elements that, in addition to general criteriological or nomological elements, make up comprehensive diagnosis are really narrative in this sense then they do add something genuinely distinct without risk to their validity. They add a rational normative form of understanding that has a different underlying logic to explanation by subsumption under natural laws. But while they furnish accounts that can be tailored to individual subjects and help to outline individual human subjectivity, this is not to deny their implicit generality. The concepts that form narrative accounts can be applied in different ways to different subjects providing that the narratives so formed are shaped in recognisable rational patterns.

\section{Conclusion}

Mental health care raises as many conceptual as empirical issues. One aspect that deserves closer scrutiny is the nature of clinical judgement of which one facet is the call for idiographic elements in comprehensive diagnosis. But there are many others. Indeed the growing success of psychiatry in understanding both the mind and brain, as well as changing political pressures on mental health care, will make this more rather than less true. That is why a closer, and two-way, relation between philosophy and psychiatry - exemplified in teaching programmes /such as the UCLan distance learning programme in Philosophy and Mental Health $^{3}$ ) - is much to be welcomed.

\section{Tim Thornton}

Professor of Philosophy and Mental Health Institute for Philosophy, Diversity and Mental Health University of Central Lancashire

Preston

UK

1. Windelband W. History and natural science. History and Theory 1980; 19: 169-185.

2. McDowell J. Functionalism and anomalous monism. In: LePore E, Mclaughlin BP, eds. Actions and Events: Perspectives on the Philosophy of Donald Davidson. Oxford: Blackwell, 1985: 387-398

3. www.uclan.ac.uk/philosophyandmentalhealth 